\title{
Kidney Diseases and COVID-19 Pandemic - A Review Article
}

\author{
Tarek Samy Abdelaziz* \\ Department of Internal Medicine, Division of Renal Medicine, Kasr-Alainy Hospitals, Cairo University, Cairo, Egypt
}

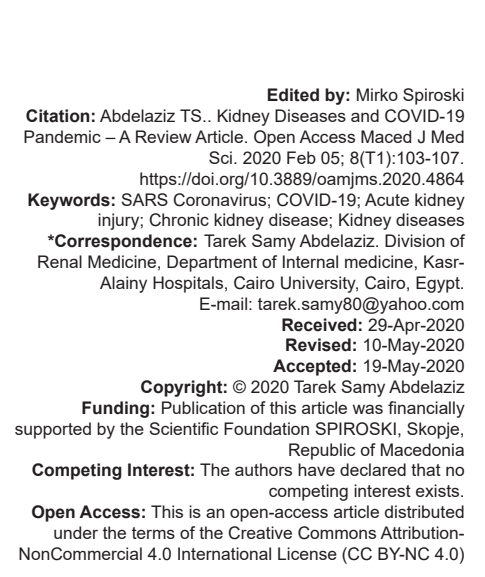

Abstract

In December 2019, first cases of a novel coronavirus were identified in Wuhan, China. A state of global pandemic was shortly declared among a very rapid contagious spread of the virus. The causative virus was identified as the SARS-CoV-2 viruses and is genetically related to the previous SARS outbreak in 2003. The virus causes a wide clinical spectrum from mild flu-like symptoms to adult respiratory distress syndrome. Kidney involvement has been reported in several reports in patients with various degrees of severity of SARS-CoV-2 infection. As knowledge is evolving, the accurate incidence of acute kidney injury (AKI) is not known. Many questions are yet to be answered regarding the effect of epidemiological variables and comorbidities on the occurrence of AKI. Some reports have observed the occurrence of hematuria and proteinuria in a percentage of infected patients. Moreover, chronic kidney disease has not been found, in some reports to add to the adverse outcomes, an aspect that merits further exploration. Patients on regular hemodialysis may be vulnerable to coronavirus infection due to the lower status of immunity and the need for frequent attendance at health-care facilities. Due to the previous factors, prevention and mitigation of the SARS-CoV-2 virus, in this vulnerable population, constitutes a major challenge.

\section{Introduction}

\section{Origin and epidemiology}

SARS-CoV-2 is a novel mutant of the coronavirus family that is causing the most recent and ongoing pandemic. The coronavirus is thought to have been transmitted at first instance from bats to humans. A wet wild animal market is likely considered to be the primary focus. The first cases of human infections were then reported in the city of Wuhan, the capital of Hubei Province of China. This was followed by widespread of the pandemic to many countries around the globe. Until the time of writing this paper (April 6, 2020), the number of infected people around the globe exceeded $1,300,000$ patients with mortality over 74,000 [1].

\section{Pathogenesis}

COVID-19 primarily targets the respiratory system, causing a wide clinical spectrum from mild symptoms to adult respiratory distress syndrome. The pathogenesis is mediated in severe cases through the so-called cytokine storm (Figure 1). This involves the secretion of large amounts of pro-inflammatory cytokines and chemokines including IL8, IL 6, IL9, IL10, and many others. Pathogenic mechanisms in the kidneys are not fully elucidated, but the suggested mechanisms are through attachment of the virus to
ACE2 receptors. Excess secretion of cytokines leads to multiorgan failure in a percentage of patients, including acute kidney injury (AKI), through tissue hypoxia [2].

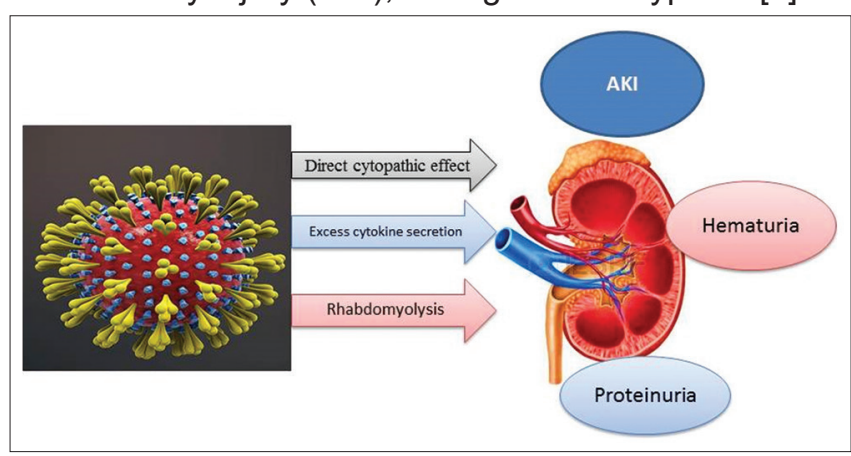

Figure 1: Renal complications of SARS-CoV-2 virus

\section{Chronic kidney disease (CKD) as a predisposing comorbidity in the event of COVID-19}

A number of studies have analyzed patients' comorbidities. The prevalence of CKD was variable across studies, ranging from 0.7 to $6.5 \%$ [3]. Only one cohort of 710 patients reported that $40 \%$ of patients had CKD in the form of deranged kidney function, hematuria, or proteinuria [4]. Most studies that used regression analysis to predict poor outcome have not identified chronic kidney disease as an important prognostic factor [5]. The lack of stratification of CKD among the prognostic factors of SAR-CoV-2 denotes that patients with CKD are not particularly at a higher risk of SAR-CoV-2 infection [3], [5]. 
Patients with end-stage renal disease (ESRD) on hemodialysis have a lower immunity status to various types of infections. The impact of COVID-19 infection on hemodialysis patients merits further investigation. In a study of 230 patients on hemodialysis in Wuhan, during the COVID-19 outbreak, 37 patients and 4 health-care providers became infected. The clinical features of hemodialysis patients reported in this cohort were mild. Seven deaths occurred among dialysis patients during the outbreak; however, causes of death were not attributed to COVID-19 [6].

\section{AKI as a complication of COVID-19}

Acute kidney injury is a common complication of several infections. In the previous SARS-CoV-2 outbreak in 2003, the incidence of AKI was as low as $6 \%$ [2], [7]. Nevertheless, AKI case fatality rate was high [8]. As for SARS-CoV-2, the exact incidence of AKI is not well known. Cohorts that reported AKI incidence are summarized in Table 1 [9],[10], [11], [12], [13], [4], [3], [14], [15], [16], [17], [18], [19], [20], [21], [5].

There is heterogeneity among studies regarding the reported incidence of AKI. This may be attributed to inconsistencies in applying AKI definitions or due to genetic variability that merits further studies.

Some reports have shown that the incidence of AKI is significant, while others report that the incidence is marginal. Guan et al. have shown, in a large cohort of confirmed COVID-19 cases that the prevalence of AKI was as low as $0.5 \%$. This increased in patients with severe COVID-19 to $2.9 \%$ [5]. In other cohorts, the incidence of AKI in confirmed cases of COVID-19 was higher.

In two cohorts, the reported incidence of AKI was notably higher. In a cohort of 193 patients, the overall incidence of AKI was $28 \%$ and the incidence in severe cases was $66 \%$ [18]. In another cohort of 191 patients, the incidence of AKI in non-survivors was $50 \%$ [19]. In the study by Hu et al., AKI was present in 17 of all 323 patients (5.3\%); however, the incidence of AKI in patients with critical COVID-19 was $38.5 \%$. Furthermore, in this cohort, most patients who had AKI (14 out of 17) had unfavorable outcomes [17].

Interestingly, one retrospective study of 116 patients showed that the changes in kidney function throughout the disease course were subtle [13]; this study included five patients on maintenance hemodialysis, all of whom had severe disease but survived. Despite the subtle changes in kidney function, none of patients in this cohort met the defining criteria for AKI, including seven deaths that were reported [13]. This report concluded that AKI and other kidney diseases are not of paramount clinical significance in patients with COVID-19 [13].

During a previous SARS outbreak in 2003, a study of postmortem kidney biopsies examined using electron transmission microcopy, failed to detect any viral particles in kidney tissues. This finding supports the theory that most of the kidney pathogenesis in the earlier SARS outbreak was in the context of multiorgan failure. The pathogenesis of AKI may be multifactorial. Suggested mechanisms are direct cytopathic effects on kidney tissues, as denoted by the retrieval of the viral RNA from urine samples [4]. The direct cytopathic effect of COVID-19 on kidneys is now more evident, as it has been shown that there is overexpression of both ACE2 receptors and a cleavage spike protein in podocytes and proximal tubular cells [22]. This experimental evidence is of paramount importance and can explain proteinuria in patients with COVID-19. Interestingly, the latter experiment reports variable expression of cleaved $S$ protein such that there is low expression in the Chinese race as compared to Caucasians. Important pathological evidence was reported by Diao et al. The pathology team managed to confirm the visualization of the SARS-CoV-2 viral particles in the renal tubular cells of postmortem kidney biopsies [23], [24]. The difference in kidney tropism between SARS-CoV and SARS-CoV-2 may be attributed to the affinity to ACE2 receptors in the kidneys.

Tissue hypoxia, in the context of massive cytokine secretion, is a key renal pathogenic mechanism. Rhabdomyolysis and raised creatinine kinase have been observed in a few cases [5]. It was also noticed in one cohort that AKI occurred later to acute cardiac injury, suggesting a temporal relationship between cardiac injury and $\mathrm{AKI}$ and the possible occurrence of cardiorenal syndrome [19]. In a recent single case report, collapsing variant of focal segmental glomerulosclerosis was diagnosed in renal biopsy of African-American woman, who tested positive to COVID-19. The patient presented with confusion and rapidly deteriorating kidney function, she improved markedly with the initiation of dialysis [25].

\section{Hematuria and proteinuria}

In the largest prospective cohort of kidney diseases in COVID-19, it was found that hematuria occurred in $26 \%$ of patients and proteinuria occurred in about $43 \%$ [4]. Large prevalence of proteinuria could be explained by the finding of the above-mentioned experimental study that showed expression of ACE 2 receptors in podocytes and proximal tubular cells [22]. However, quantification of proteinuria, using $24 \mathrm{~h}$ urinary collection or protein to creatinine ratio, was not done within the investigation battery. Kidney biopsy has not been attempted in any patient. In this prospective report, the presence of hematuria or proteinuria signaled poor outcome, as measured by in-hospital mortality.

\section{Effect of dialysis modalities on survival in patients infected with COVID-19}

Continuous renal replacement therapy (CRRT) is a modality of dialysis that implies increasing the clearance of solutes through convection, diffusion, ultrafiltration, 


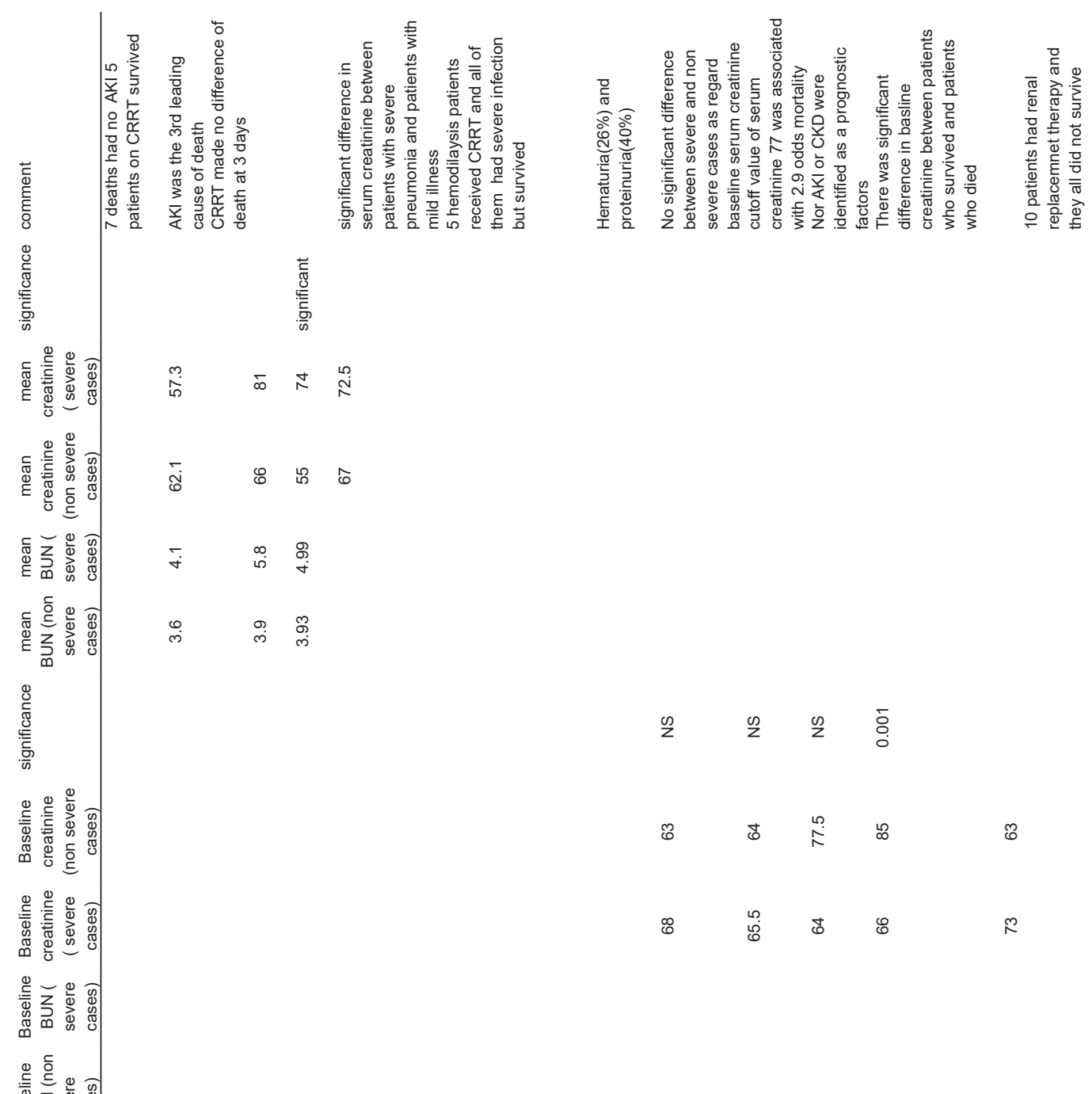

II

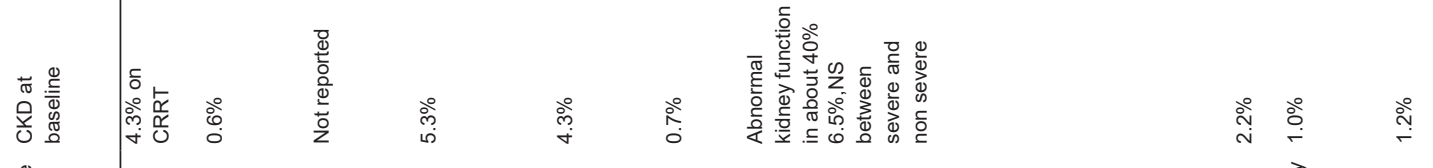

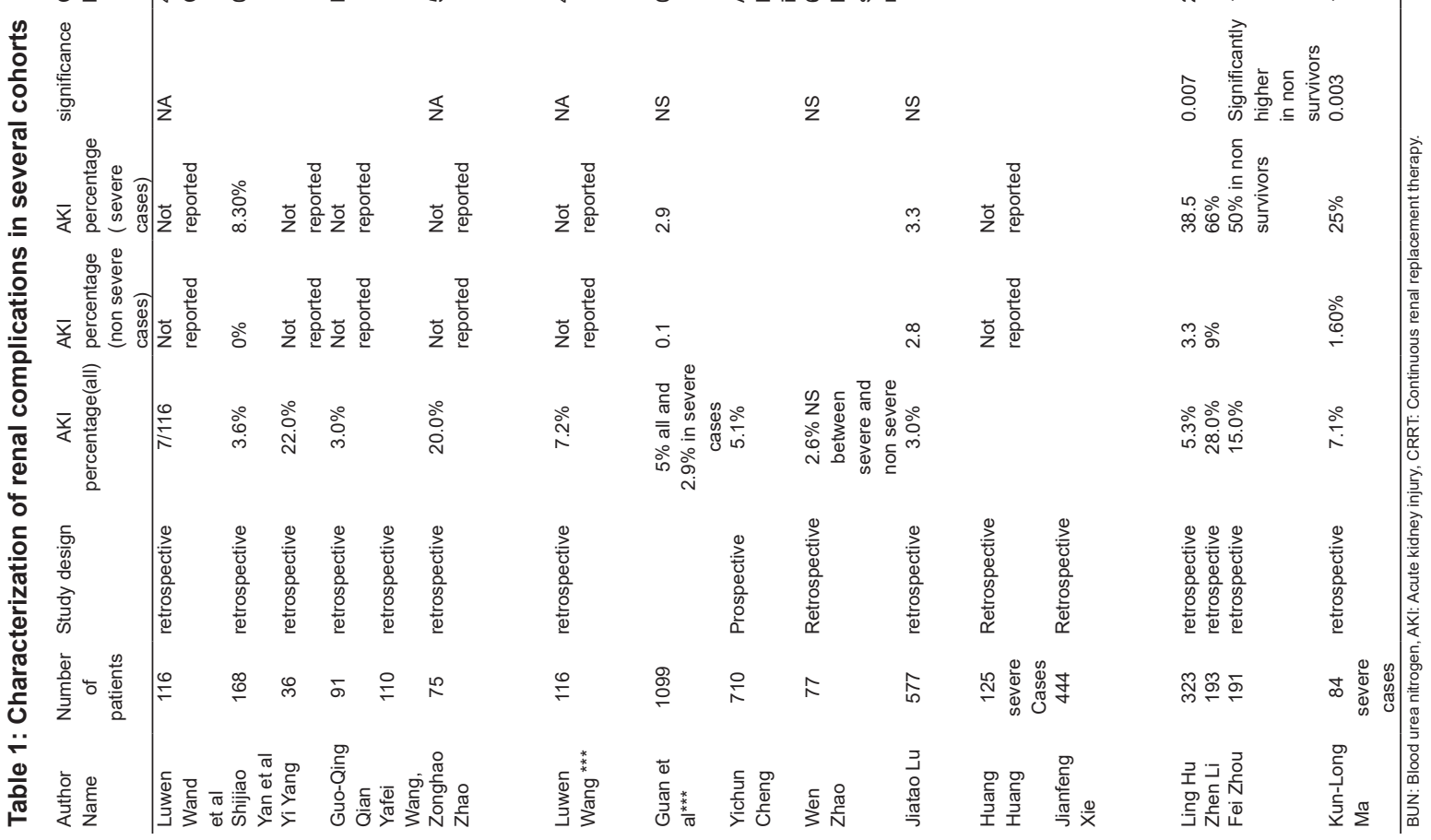


and adsorption. The modality has benefits in critically ill patients, including the removal of septic toxins in addition to correction of the uremic status. There is accumulating evidence that critically ill patients who develop AKI may have lower mortality if they are treated using CRRT [26].

As knowledge is evolving about the SARSCoV-2 virus, the benefit of CRRT in the management of critically ill patients with COVID-19 is much less clear. One retrospective study was conducted in China on 36 confirmed COVID-19 cases who have been admitted to the intensive care unit [10]. All patients were mechanically ventilated; the aim was to compare the effect of CRRT as compared to conventional dialysis. Patients were followed up for an average time of 10.4 days. The mean serum creatinine was slightly higher in patients who received CRRT than in patients who did not receive CRRT $(94.5 \mathrm{mmol} / \mathrm{L}$ vs. $72 \mathrm{mmol} / \mathrm{L}$, $p=0.017$ ). There was a marginally a favorable effect of CRRT in terms of adjusted mortality (54.4\% in CRRT group vs. $78 \%$ in the conventional hemodialysis group).

On the contrary, another analysis of risk factors and survival in critically ill patients found that non-survivors received more treatment with CRRT than survivors.

Another study of 101 case fatalities in China, five cases had CRRT. Two patients died within 3 days and three patients died after 3 days. The mean baseline serum creatinine was $139.8 \mu \mathrm{mol} / \mathrm{L}$ [27]. In another large retrospective analysis by Guan et al., nine patients were treated with CRRT, eight of whom died suggesting that CRRT had no mortality benefit [5]. In a cohort of 191 patients, 10 patients received renal replacement therapy, and all did not survive, suggesting that renal replacement therapy in severe cases of COVID-19 may not have any survival benefit [19].

\section{Renal-specific mortality due to COVID-19}

The leading causes of mortality in COVID-19infected patients are sepsis and ARDS. This has been observed in several cohorts. A large prospective study showed that the development of $\mathrm{AKI}$ in patients infected with COVID-19 was associated with four-fold increase in the mortality [4]. In other reports, renal-specific causes were not the most common or the second most common of mortality in COVID-19. In a retrospective study of 101 non-surviving COVID-19 patients, the incidence of AKI was $23 \%$, there was no significant difference between patients who died within 3 days and patients who died later with regard to $\mathrm{AKI}$ incidence ( $25 \%$ vs. $21 \%, \mathrm{p}=0.611)$ [27]. In this cohort, AKI was the $3^{\text {rd }}$ leading cause of death after respiratory and cardiovascular causes. In a singlecentered study in China, chronic kidney disease was present in 7 out of 323 patients (2\%). Four patients had severe disease while the other three patients had non severe disease. Elevation of BUN > $88 \mathrm{mmol} / \mathrm{L}$ was associated with a two-fold increase in the chance of poor clinical outcomes. Baseline serum creatinine of
$<88 \mathrm{mmol} / \mathrm{L}$ was associated with $63 \%$ reduction in the development of poor outcomes. In another report of 82 non-surviving patients with confirmed COVID-19, the AKI percentage was 31\% [28].

\section{Prevention and mitigation of COVID-19 among dialysis patients}

Until the time of writing this paper, there is no consensus or formal approval of any medication for COVID-19. This fact mandates the exhaustion of all measures to prevent the transmission of infection. In this respect, the Centers for Disease Control and Prevention (CDC) has issued an interim guideline for hemodialysis centers. The guideline emphasizes the importance of early recognition and isolation of cases while attending their scheduled sessions [29]. This mandates treating confirmed cases of COVID-19 hemodialysis in designated rooms with droplet infection prevention precautions; patients with confirmed of suspected COVID-19 should be separated by 6 feet distance. The instructions for hemodialysis patients should be centralized around reporting any new symptoms of fever or cough. Patients should be instructed on the proper use of face masks and using tissues when sneezing or coughing to prevent spread of infections [30], [29]. There is an anticipated extraordinary strain on hemodialysis facilities. In parallel, there are a number of suggestions to match the resources. These practical suggestions aim at reduction of the strain on hemodialysis units [29].

\section{Summary and Conclusion}

SARS-CoV-2 (COVID-19) virus pandemic constitutes a global health threat. The disease spectrum caused by the virus flu-like symptoms to adult respiratory distress syndrome. Kidney involvement in COVID-19 has been reported in previous cohorts. Acute kidney injury is a complication of COVID-19, either as part of multiorgan failure caused by excess cytokine production or through direct cytopathic effect on renal tissue. Overexpression of ACE2 receptors in podocytes and proximal tubular cells has been observed in patients with COVID-19. Other renal manifestations include proteinuria and hematuria. The true incidence and outcome of AKI in COVID-19 is not entirely clear and merits further studies. Continuous renal replacement therapy (CRRT) benefits in case of AKI are controversial. Patients on hemodialysis may be at increased risk of COVID-19 due to the nature of renal replacement therapy that requires 3 times weekly attendance at dialysis facilities. This necessitates the application of meticulous measures to prevent and mitigate the outbreak in patients on hemodialysis. 


\section{References}

1. World Health Organization. Coronavirus Disease (COVID2019) Situation Reports. Available from: https://www.who. int/emergencies/diseases/novel-coronavirus-2019/situationreports. [Last accessed on 2020 Apr 06].

2. Perico L, Benigni A, Remuzzi G. Should COVID-19 concern nephrologists? Why and to what extent? The emerging impasse of angiotensin blockade. Nephron. 2020;144(5):213-21. https:// doi.org/10.1159/000507305

3. ZhaoW,YuS,ZhaX,WangN,PangQ,LiT,etal.Clinicalcharacteristics and durations of hospitalized patients with COVID-19 in Beijing: A retrospective cohort study. MedRxiv. 2020;2020:20035436. https://doi.org/10.1101/2020.03.13.20035436

4. Cheng Y, Luo R, Wang K, Zhang M, Wang Z, Dong L, et al. Kidney disease is associated with in-hospital death of patients with COVID-19. Kidney Int. 2020;97(5):829-38. https://doi. org/10.1016/j.kint.2020.03.005

PMid:32247631

5. Guan W, Ni Z, Hu Y, Liang W, Ou C, He J, et al. Clinical characteristics of coronavirus disease 2019 in China. N Engl J Med. 2020;382(18):1708-20. https://doi.org/10.1056/ NEJMoa2002032

6. Naicker S, Yang C, Hwang S, Liu B, Chen J, Jha V. The novel coronavirus 2019 epidemic and kidneys. Kidney Int. 2020;97(5):824-8. https://doi.org/10.1016/j.kint.2020.03.001 PMid:32204907

7. Chu KH, Tsang WK, Tang CS, Lam MF, Lai FM, To KF, et al. Acute renal impairment in coronavirus-associated severe acute respiratory syndrome. Kidney Int. 2005;67(2):698-705. https:// doi.org/10.1111/j.1523-1755.2005.67130.x

PMid:15673319

8. Kwan BC, Leung C, Szeto C, Wong VW, Cheng Y, Yu AW, et al. Severe acute respiratory syndrome in dialysis patients. J Am Soc Nephrol. 2004;15(7):1883-8.

PMid: 15213277

9. YanS, SongX, LinF, ZhuH, WangX, LiM, etal. Clinical characteristics of coronavirus disease 2019 in Hainan, China. MedRxiv. 2020;2020:20038539. doi.org/10.1101/2020.03.13.20035436

10. Yang Y, Shi J, Ge S, Guo S, Xing X, Wang Y, et al. Effect of continuous renal replacement therapy on all-cause mortality in COVID-19 patients undergoing invasive mechanical ventilation: A retrospective cohort study. MedRxiv. 2020;2020:3. https://doi. org/10.1101/2020.03.16.20036780

11. Wang Y, Zhou Y, Yang Z, Xia D, Geng S. Clinical characteristics of patients with severe pneumonia caused by the 2019 novel coronavirus in Wuhan, China. MedRxiv. 2020;2020:20029306. https://doi.org/10.1101/2020.03.02.20029306

12. ZhaoZ, Xie J, Yin M, Yang Y, HeH, Jin T, etal. Clinical and laboratory profiles of 75 hospitalized patients with novel coronavirus disease 2019 in Hefei, China. MedRxiv. 2020;2020:20029785. https://doi.org/10.1101/2020.03.01.20029785

13. Wang L, Li X, Chen H, Yan S, Li D, Li Y, et al. Coronavirus disease 19 infection does not result in acute kidney injury: An analysis of 116 hospitalized patients from Wuhan, China. Am J Nephrol. 2020;51(5):343-8. https://doi.org/10.1159/000507471

14. Lu J, Hu S, Fan R, Liu Z, Yin X, Wang $Q$, et al. ACP risk grade: A simple mortality index for patients with confirmed or suspected severe acute respiratory syndrome coronavirus 2 disease (COVID-19) during the early stage of outbreak in Wuhan, China. MedRxiv. 2020;2020:20025510. https://doi. org/10.1101/2020.02.20.20025510

15. Huang H, Cai S, Li Y, Li Y, Fan Y, Li L, et al. Prognostic factors for COVID-19 pneumonia progression to severe symptom based on the earlier clinical features: A retrospective analysis. MedRxiv. 2020;2020:20045989. https://doi. org/10.1101/2020.03.28.20045989

16. Xie, J, Hungerford D, Chen H, Abrams ST, Li S, Wang G, et al. Development and external validation of a prognostic multivariable model on admission for hospitalized patients with COVID-19. MedRxiv. 2020;2020:20045997. https://doi. org/10.1101/2020.03.28.20045997

17. Hu L, Chen S, Fu Y, Gao Z, Long H, Ren H, et al. Risk factors associated with clinical outcomes in 323 COVID-19 patients in Wuhan, China. MedRxiv. 2020;2020:20037721.

18. Li Z, Wu M, Yao J, Guo J, Liao X, Song S, et al. Caution on kidney dysfunctions of COVID-19 patients. MedRxiv. 2020;2020:20021212.

19. Zhou F, Yu T, Du R, Fan G, Liu Y, Liu Z, et al. Clinical course and risk factors for mortality of adult inpatients with COVID-19 in Wuhan, China: Aretrospective cohort study. Lancet. 2020;395(10229):105462. https://doi.org/10.1016/s0140-6736(20)30566-3

20. Ma K, Liu Z, Cao C, Liu M, Liao J, Zou J, et al. COVID-19 Myocarditis and severity factors. An adult cohort study. MedRxiv. 2020;2020:20034124.

21. Qian G, Yang N, Ding F, MaAH, Wang Z, Shen Y, et al. Epidemiologic and clinical characteristics of 91 hospitalized patients with COVID19 in Zhejiang, China: A retrospective, multi-centre case series. QJM. 2020;2020:hcaa089. https://doi.org/10.1093/qjmed/hcaa089 PMid:32181807

22. Pan $X, X u D$, Zhang $H$, Zhou W, Wang L, Cui X. Identification of a potential mechanism of acute kidney injury during the COVID-19 outbreak: A study based on single-cell transcriptome analysis. Intensive Care Med. 2020;2020:1-3. https://doi. org/10.1007/s00134-020-06026-1

PMid:32236644

23. DiaoB, Wang C, Wang R, FengZ, Tan Y, Wang H, etal. Humankidney is a target for novel severe acute respiratory syndrome coronavirus 2 (SARS-CoV-2) infection. MedRxiv. 2020;2020:20031120. https://doi.org/10.1101/2020.03.04.20031120

24. Wan Y, Shang J, Graham R, Baric RS, Li F. Receptor recognition by the novel coronavirus from Wuhan: An analysis based on decade-long structural studies of SARS coronavirus. J Virol. 2020;94(7):e00127-20. https://doi.org/10.1128/jvi.00127-20 PMid:31996437

25. Larsen CP, Bourne TD, Wilson JD, Saqqa O, Sharshir MA. Collapsing glomerulopathy in a patient with coronavirus disease 2019 (COVID-19). Kidney Int Rep. 2020;5:935-9. https://doi. org/10.1016/j.ekir.2020.04.002 PMid:32292867

26. Putzu A, Fang MX, Berto MB, Belletti A, Cabrini L, Cassina T, et al. Blood purification with continuous veno-venous hemofiltration in patients with sepsis or ARDS: A systematic review and metaanalysis. Minerva Anestesiol. 2017;83(8):867-77. PMid:28607338

27. Shi Q, Zhao K, Yu J, Feng J, Zhao K, Zhang X, et al. Clinical characteristics of 101 non-surviving hospitalized patients with COVID-19: A single center, retrospective study. MedRxiv. 2020;2020:20031039.

28. Zhang B, Zhou X, Qiu Y, Feng F, Feng J, Jia Y, et al. Clinical characteristics of 82 death cases with COVID-19. MedRxiv. 2020;2020:20028191.

29. Kliger AS, Silberzweig J. Mitigating risk of COVID-19 in dialysis facilities. Clin J Am Soc Nephrol. 2020;15(5):707-9. https://doi. org/10.2215/cjn.03340320

PMid:32198130

30. Basile C, Combe C, Pizzarelli F, Covic A, Davenport A, Kanbay $\mathrm{M}$, et al. Recommendations for the prevention, mitigation and containment of the emerging SARS-CoV-2 (COVID-19) pandemic in hemodialysis centres. Nephrol Dial Transplant. 2020;35(5):737-41. https://doi.org/10.1093/ndt/gfaa069 